\title{
A Direction-Sensitive Dark Matter Search Experiment (NEWAGE)
}

\author{
Hironobu Nishimura*, Kaori Hattori, Chihiro Ida, Satoru Iwaki, Shigeto Kabuki, \\ Hidetoshi Kubo, Shunsuke Kurosawa, Kentaro Miuchi, Atsushi Takada, Michiaki \\ Takahashi, Toru Tanimori and Kazuki Ueno \\ Cosmic-Ray Group,Department of Physics, Faculty of Science, Kyoto Univercity \\ E-main:nisimuradcr.scphys.kyoto-u.ac.jp
}

\begin{abstract}
Weakly interacting massive particles (WIMPs) are very plausible candidates of dark matter. We developed a three-dimensional gaseous tracking device, and performed a direction-sensitive dark matter search in a surface laboratory. By using 150 torr carbon-tetrafluoride $\left(\mathrm{CF}_{4} \mathrm{gas}\right)$, we obtained a sky map drawn with the recoil directions of the carbon and fluorin e nuclei, and set the first limit on the spin-dependent WIMP (Weakly Interacting Massive Particles)-proton cross section by a direction-sensitive method.

In this paper, we describe the detector performance and report on the direction-sensitive WIMPsearch experiment in a surface laboratory.
\end{abstract}

Identification of dark matter 2008

August 18-22, 2008

Stockholm, Sweden

\footnotetext{
* Speaker.
} 


\section{Introduction}

Most dark matter search experiments are designed to measure only the energy transferred to the nucleus through WIMP-nucleus scatterings [1- 10]. Because the amplitude of an annual modulation of the energy spectrum, which is distinct signals of WIMPs of these experiment, is only a few $\%$ of the rate, positive signals of WIMPs are very difficult to detect. On the other hand, the motion of the solar system with respect to the galactic halo is considered to provide a much larger asymmetry in the direction-distribution of the WIMP velocity observed at Earth. A detection method of a positive signature of WIMPs by measuring the directions of the nuclear recoils have been studied [5]. Gaseous detectors are one of the most appropriate devices to detect this "WIMPwind". Since former studies suggest to perform the WIMP-search both via spin-independent (SI) and spin-dependent(SD) interactions [11], we propose a SD-sensitive WIMP-wind search, named NEWAGE (New generation WIMP-search with an Advanced Gaseous tracking device Experiment) [12].

\section{Detector}

We developed a small-volumed $\mu$-TPC. A $\mu$-TPC is a gaseous time projection chamber with a micro pixel chamber ( $\mu$-PIC) readout. A $\mu$-PIC is a gaseous two-dimensional position-sensitive detector manufactured by printed circuit board technology, which is originally developed in our laboratory. We developed a large-sized $\left(31 \times 31 \mathrm{~cm}^{2}\right) \mu$-PICs[13], and subsequently developed a $\mu$-TPC with a detection volume of $23 \times 28 \times 30 \mathrm{~cm}^{3}[14]$. We use the $\mu$-TPC with 150 torr $\mathrm{CF}_{4}$ gas ( $9.0 \mathrm{~g}$ effective mass) and total gas gain of 2300 , which is high enough to maintain the detection efficiency of recoil nucleus. The nuclear detection efficiency was $40 \%$ at $100 \mathrm{keV}$ and the $90 \%$ C.L. upper limit of the gamma-ray rejection factor was $1.5 \times 10^{-4}$. The position resolution, which was measured using the same method as described in our previous work[14], was $0.8 \mathrm{~mm}$ (RMS). The details of these performance were described in Ref. [15].

\section{Surface measurement result}

A dark matter search measurement was performed in a surface laboratory at Kyoto University (Lat. $35^{\circ} 2^{\prime}$ N, Long. $135^{\circ} 34^{\prime}$ E) from November 1st, 2006 to November 27th, 2006. The total live time was 16.71 days and the exposure was $0.151 \mathrm{~kg}$ days. The sum of the calibration and maintenance time was 7.51 days, and the dead time due to the data acquisition was 1.0 days. The obtained spectrum is shown in Fig.1. We initially derived the limits of the WIMP-proton spindependent cross section only from the spectrum (conventional method). We next performed a direction-sensitive analysis [15]. We created a sky map of the north sky with the direction of the three dimensional nuclear recoil tracks. Because we did not detect the sense of each track, the map was restricted to half of the sky and the southern part of the sky was folded. We plotted every nuclear event in Fig. . The larger maker, labeled "DM expected", is the direction of the solar motion or Cygnus from which the WIMP-wind is expected. We calculated $\theta$, the angle between the WIMP-wind direction and the recoil direction for each event, and drew the $\cos \theta$ distribution shown in Fig. We compared this measured $\cos \theta$ distribution with the expected WIMP-signal. 
The best fitted function of WIMP-signals of the data was rejected at the $90 \%$ confidence level by a $\chi^{2}$ test. And we set the upper limits shown with thick-solid line (labeled "direction-sensitive") in Fig. (4. It was demonstrated that the direction-sensitive method gave a limit slightly better than the conventional spectrum-only method. This is first limit set by a dark matter search experiment with a gaseous tracking device. As for the spin-dependent limit, this is the first result by a directionsensitive experiment.

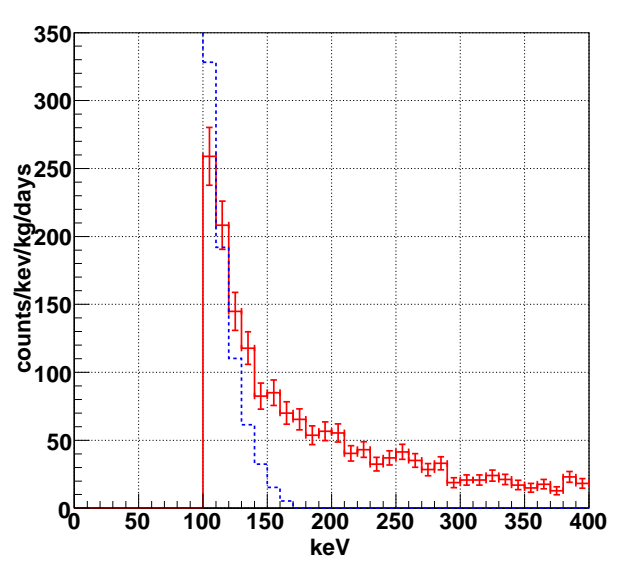

Figure 1: Measured energy spectrum (solid red histogram)[15], where the detection efficiency is taken into account and expected energy spectrum with assuming $\sigma_{\text {WIMP-p }}=1.82 \times 10^{4} \mathrm{pb}$ (dashed blue histogram).

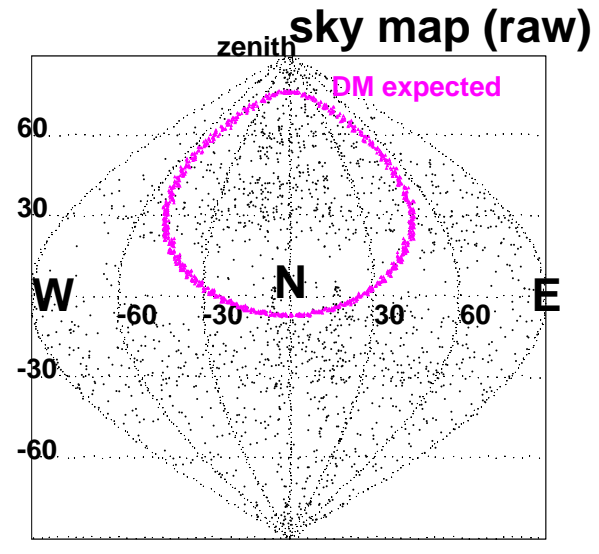

Figure 2: Obtained sky map of north sky. The recoil directions of the fluorine and carbon nuclei are used. The thick line indicates the direction of the solar motion (direction of WIMP-wind). 15]

\section{Future work and Underground experiment}

As a next step, we started an underground measurement in Kamioka Observatory located at 2700 m.w.e. underground $\left(36^{\circ} 25^{\prime} \mathrm{N}, 137^{\circ} 18^{\prime} \mathrm{E}\right)$ from January, 2007 to check the stability of the performance of the detector for a long operation and to measure the background from the detector components. The detector was operated stably without serious problems from January to August, 2007, while we got the exposure of $0.23 \mathrm{~kg}$ days. We estimated internal background caused by the radioactive impurity which must be eliminated. We will improve the purity for low background measurement and make a larger detector with lower energy threshold with lower pressure gas for a next generation WIMP search.

\section{Acknowledgment}

This work was partially supported by Grant-in-Aids for KAKENHI(19684005) of Young Scientist(A), JSPS Fellows, and Global COE Program "The Next Generation of Physics, Spun from Universality and Emergence" from Ministry of Education, Culture, Sports, Science and Technology (MEXT) of Japan. 

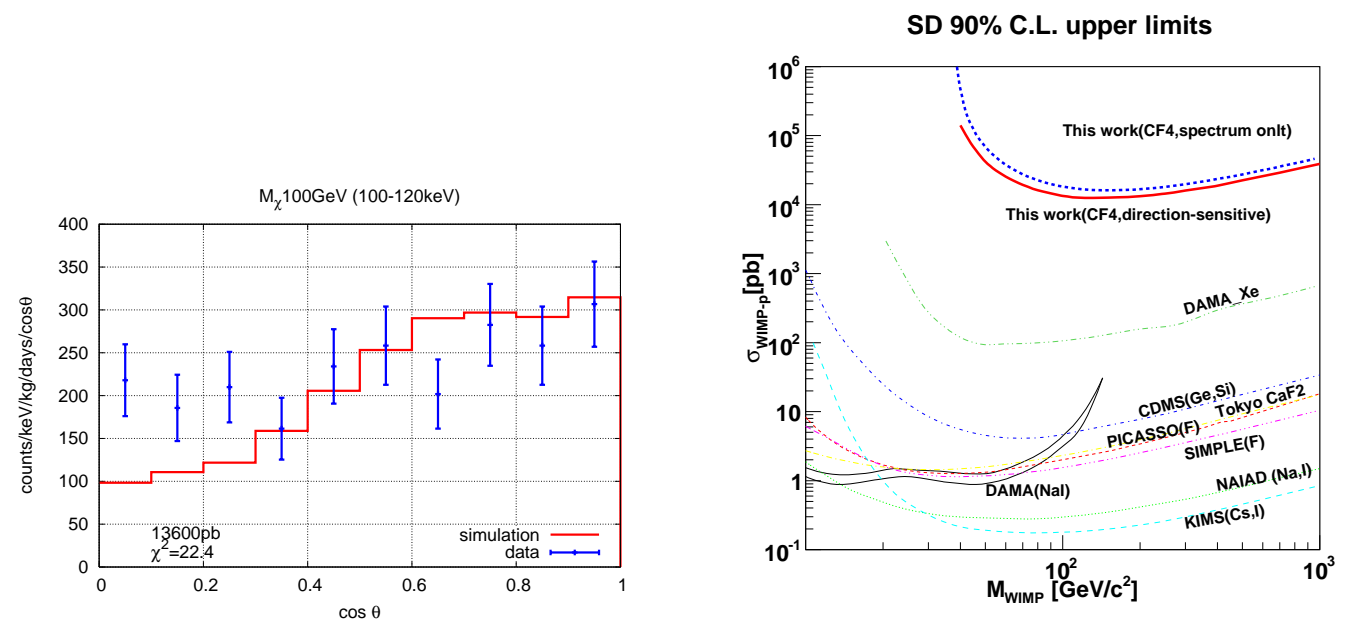

Figure 3: Measured (with error bars) and expected Figure 4: $90 \%$ C.L. upper limits on the WIMP(histogram) distributions of the angle between the proton spin-dependent cross section. The thick recoil direction and the WIMP direction. The ex- solid and dotted lines show the limits obtained pected histogram is that with $100 \mathrm{GeV}$ of WIMPs with and without the direction information, mass , 100-120 keV bin and $1.36 \times 10^{4} \mathrm{pb}$. 15 respectively[15]. Limits from other experiments (DAMA(Xe)[五], DAMA(NaI)[3], NAIAD[4], Tokyo $\left(\mathrm{CaF}_{2}\right)[$ [], $\quad$ SIMPLE[7], PICASSO[8], CDMS[9] and KIMS [10] ) are shown for comparison.

\section{References}

[1] P.Beli et al., Nuovo Cimento C 15 (1992) 475.

[2] R. Bernabei et al., Phys. Lett. B 436 (1998) 379.

[3] R. Bernabei et al., Phys. Lett. B 480 (2000) 23.

[4] Uk Dark Matter Collaboration: Phys. Lett. B 616 (2005) 17.

[5] H. Sekiya et al., Proceedings of the Fourth International Workshop on "The Identification of Dark Matte", Edinburgh, UK, 2004, N.J.C.Spooner, V.Kudryavtsev(Eds.), World Scientific, 2005, p. 378.

[6] Y. Shimizu et al., Phys. Lett. B 633 (2006) 195.

[7] T. A. Girard et al., Phys. Lett. B 621 (2005) 233.

[8] PICASSO Collaboration, Phys. Lett. B 624 (2005) 186.

[9] CDMS Collaboration, Phys. Rev. D 73 (2006) 011102.

[10] KIMS Collaboration, Phys. Rev. Lett. 99 (2007) 091301.

[11] T.A.Girard and F.Giuliani, Phys. Rev. D 75 (2007) 043512.

[12] T. Tanimori et al., Phys. Lett. B 578 (2004) 241.

[13] A.Takada et al., Nucl. Instrum. Methods. A 573 (2007) 195.

[14] K. Miuchi et al., Nucl. Instrum. Methods. A 576 (2007) 43.

[15] K. Miuchi et al., Phys. Lett. B 654 (2007) 58. 